\title{
Determination of charge-carrier transport in organic devices by admittance spectroscopy: Application to hole mobility in $\alpha$-NPD
}

\author{
N. D. Nguyen* and M. Schmeits \\ Institute of Physics, University of Liege, B-4000 Liege, Belgium
}

H. P. Loebl

Philips Research Laboratories, Weisshausstrasse 2, D-52066 Aachen, Germany

(Received 9 August 2006; revised manuscript received 11 November 2006; published 6 February 2007)

Hole mobility in $N, N^{\prime}$-diphenyl- $N, N^{\prime}$-bis(1-naphtylphenyl)-1, $1^{\prime}$-biphenyl-4, $4^{\prime}$-diamine $(\alpha$-NPD) is evaluated by electrical characterization in the ac regime. The frequency-dependent complex admittance and impedance of the structure consisting of the organic layer, grown by thermal evaporation, sandwiched by indium tin oxide and aluminum electrodes, are measured as functions of the applied dc voltage. The capacitance response shows negative values for frequencies below a characteristic value depending on the bias and ranging from $0.1 \mathrm{~Hz}$ up to $20 \mathrm{~Hz}$. It increases with the modulation frequency and reaches a peak, the magnitude and position of which are functions of the applied voltage. For higher frequencies, a minimum can be observed before the capacitance increases again up to a constant value. A final decreasing occurs at frequency of $4 \times 10^{6} \mathrm{~Hz}$. The analysis of the experimental data is performed by a detailed theoretical study of the steady-state and smallsignal electrical characteristics of the device. Numerical calculations are based on the solution of the basic semiconductor equations for the system consisting of two electrodes connected by the semiconducting channel formed by the organic layer. The description explicitly includes a continuous distribution of trap density of states and a field-dependent carrier mobility. The spatially dependent charge carrier and occupied trap concentrations, as well as the various components to the total current density, are obtained for the dc and ac regimes and are analyzed for given bias and frequency. Based on a formalism used in the study of inorganic semiconductors, the results of the simulation show that the inductive contribution to the capacitance response originates from the modulation of the hole concentration in the organic material, leading to the corresponding carrier transit time. Moreover, the low-frequency behavior of the capacitance curves could be explained by the presence of a band of defect states which modifies the charge distribution within the organic layer and the injection of electrons from the cathode. We show that the latter contribution is also responsible for the negative values of the capacitance measured below $10 \mathrm{~Hz}$. Good agreement is observed between the experimental and theoretical electrical characteristics, in particular for the differential susceptance results and the subsequent hole mobility values. Our approach can be a useful contribution for the methodology of obtaining mobilities from admittance measurements as it allows one to clarify the physical origin of the measured frequencydependent capacitance and to check for the experimental procedure. This work finally leads to the formulation of the conditions under which small-signal ac measurements can be used to determine carrier mobility in organic devices.

DOI: 10.1103/PhysRevB.75.075307

PACS number(s): 73.61.Ph, 72.80.Le, 72.20.Jv, 85.30.De

\section{INTRODUCTION}

The efficiency of charge transport in organic materials is one of the key factors which determine the overall performance of organic light-emitting diodes (OLED's). In disordered media, such as amorphous films of small molecules, the conduction mechanism involves charge transfer between localized sites, with a rate depending on temperature and applied electric field. The carrier mobility $\mu$, classically defined as the proportionality factor between drift velocity and field, is a crucial microscopic parameter describing charge transport in solids. In organic materials, the field dependence of $\mu$ usually takes the well-known Poole-Frenkel form, ${ }^{1-3}$ although some recent works suggest that the origin of such a functional dependence is due the density of charge. ${ }^{4-6}$ The measurement of carrier mobility is an important task which delivers fundamental data for the evaluation of charge transport in the device under study.

Various experimental methods have been used to perform such a measurement in thin films. The time-of-flight technique, ${ }^{7,8}$ dark current injection, ${ }^{9,10}$ and transient electroluminescence ${ }^{11,12}$ have been used to determine carrier mobility in organic materials. The common feature of these experiments is the observation of the generation or injection of charge carriers at one electrode and their drift to the opposite one. The mobility is then deduced from the delay time related to the charge transport through the organic layer. In some cases, steady-state current-voltage characterization can be employed to measure the mobility. ${ }^{13,14}$ Experiments in the ac regime can also provide a valuable tool for the investigation of transport properties in solid-state materials. ${ }^{15-20}$ In the so-called admittance spectroscopy technique, the complex impedance and admittance of the studied device are measured as functions of the modulation frequency and the applied dc voltage. By the interpretation of the frequency-dependent capacitance data, mobility in singlecarrier devices made of poly(phenylene vinylene) $)^{15,16,18,19}$ and tris(8-hydroxyquinoline) aluminum $^{17}$ was evaluated. Recently, Tsang et $a l^{20}$ have also successfully 
applied the method to a structure based on $4,4^{\prime}$, $4^{\prime \prime}$-tris $[N,-(3-$ methylphenyl)- $N$-phenylamino $]$ triphenylamine ( $m$-MTDATA). In these studies, the analysis of the smallsignal response curves was derived from an analytical formulation of the admittance obtained from the Fourier transform of the current density expression for a space-chargelimited diode. The original derivation is due to Shao and Wright, ${ }^{21}$ who assumed Ohmic injecting contacts and fieldindependent mobility, and neglected diffusion currents. In their contribution, Berleb and Brütting ${ }^{17}$ included a fielddependent mobility and the dynamics of trap states, which were eluded in other works. Admittance spectroscopy experiments were finally used in the study of bipolar carrier transport in conjugated polymers. ${ }^{19,22-24}$

In this paper, we present the results of a detailed study of the electrical characterics in the ac regime of an organic thinfilm structure. The experimentally obtained admittance data are reproduced by a theoretical model of the electrical conduction under small-signal conditions. The low-molecularweight material is treated within a description derived for inorganic semiconductor devices and usually called the driftdiffusion formalism. This framework allows the use of conduction and valence bands, assuming that free carrier transport is characterized by electron and hole mobilities. Application to structures made of organic materials may be considered as a first approximation, due to the inherent complexity of these materials. The assumption of band transport with determined mobilities for electrons and holes, which is not fully compatible with the amorphous nature of the organic layers, may thus lead to difficulties in the interpretation of the results, due to different mechanisms in the charge transport between organic and inorganic materials. However, in spite of its limitations, this approach allows a tractable description of the electrical conduction process. It is widely spread in literature and has been successfully applied to OLED's (see, e.g., Ref. 25 and references therein). The analysis starts with the classical semiconductor equations which are generally used in the description of electronic devices. ${ }^{26}$ This equation set includes Poisson's equation for the electric potential and continuity equations for holes, electrons, and traps.

The numerical simulation is performed for a structure based on $N, N^{\prime}$-diphenyl- $N, N^{\prime}$-bis(1-naphtylphenyl)$1,1^{\prime}$-biphenyl-4, $4^{\prime}$-diamine ( $\alpha$-NPD) in order to investigate the microscopic response of the organic medium to the applied ac voltage and to realize the subsequent analysis for evaluating carrier mobility. Indium tin oxide (ITO) acts as the hole injection contact (anode), and Al is taken as composition of the cathode. A set of parameters is used to characterize the organic layer, the contacts, and the experimental conditions, such as temperature, applied voltage, and modulation frequency. The expression of the current density in our approach contains both drift and diffusion terms, as well as contribution from the displacement current in the ac case. The field dependence of mobility is explicitly taken into account, and the trap states, which may be present in the real devices, are included in the calculations. In the first step of the procedure, the equations are solved for the steady-state condition. This yields the energy band diagram and the electron and hole distributions, as well as the current densities and the recombination rates. The position-dependent amplitudes of the same quantities are then obtained by smallsignal analysis. These results lead, for a given steady-state voltage, to the admittance- and impedance-versus-frequency curves which are the macroscopic quantities that can be compared to experiment. Then, a relation between the microscopic effects of the applied modulating voltage and the characteristics of the admittance spectra can be established. The philosophy that guided the present work is similar to that used for numerical studies of steady-state conduction of organic diodes which can be found in the literature. ${ }^{27-33}$ The objective is to provide a theoretical background to the interpretation of the frequency-dependent capacitance of the device. The description of the electrical conduction in the thin film allows us to explain the microscopic origin of the transit time effect and to yield final admittance curves which can be compared to experimental data. It can be considered as a useful contribution to the methodology of obtaining carrier mobility from admittance spectroscopy experiments. We show thus that the transit time effect is due to a modulation of the hole charge inside the structure. Above a frequency which is of the order of the inverse transit time, the holes no longer respond to the modulating applied voltage and the capacitance is that of a dielectric of static dielectric constant. In principle, holes are dominant in $\alpha$-NPD as their mobility is by several orders of magnitude higher than electron mobility. However, at low frequency, the negative values and the variations of the capacitance can only be explained by a combined effect of the modulation of electron concentration and the response of trap states. Minority carrier injection, determined by the barrier height at the Al cathode, leads thus to modifications in the admittance spectra that can have an influence on the determination of the hole transit time. A Gaussian distribution of trap states is included in order to reproduce the device capacitance at intermediate frequencies.

The paper is organized as follows. Details of device fabrication are given in Sec. II, as well as the experimental admittance results. Section III is devoted to a description of the basic formal developments to the numerical calculations. Results of the application to a $\alpha$-NPD-based structure are discussed in Sec. IV. The theoretical analysis starts with the case of a trap-free organic layer. The study of a device containing defect states is finally exposed and comparison with the experimental data is discussed. We summarize and conclude the paper in Sec. V.

\section{EXPERIMENT}

\section{A. Sample fabrication and measurement procedure}

The devices were fabricated by thermal evaporation of the organic material on ITO-coated substrates. Prior to deposition, substrates were thoroughly cleaned in an ultrasonic bath using subsequently detergents and de-ionized water. Finally the samples were spin dried. Organic residues were removed by a 3-min UV-ozone treatment. Then, the samples were transferred into the deposition tool via a load lock. The deposition of the $\alpha$-NPD layer was done by thermal evaporation from a heated crucible under a background pressure of $10^{-7}$ mbar. The deposition rate, on the order of $0.2 \mathrm{~nm} / \mathrm{s}$, 
and the film thickness were monitored by an Inficon quartz balance. Finally an $\mathrm{Al}$ cathode was deposited onto the organic film by thermal evaporation. The samples were packaged prior to the measurements.

The frequency-dependent impedance measurements were performed jointly with a Solatron SI 1260 impedance analyzer and a Novocontrol "alpha-A high resolution dielectric conductance impedance gain phase modular measurement system." The modulation frequency was varied from $0.1 \mathrm{~Hz}$ up to $5 \mathrm{MHz}$ with oscillation amplitude of the ac voltage set to $50 \mathrm{mV}$. A dc bias up to $10 \mathrm{~V}$ was superimposed on the ac signal.

Independently, dark current injection experiments were performed to determine the mobility of holes in the samples. The method, described in literature, ${ }^{10,34,35}$ is based on a rectangular voltage step to generate carriers in an organic thin film. We used $1-\mathrm{kHz}$ rectangular pulses made by a HP 33120A "arbitrary waveform generator." These pulses were fed into an amplifier, which allowed us to define the pulse height and offset. Due to the applied electrical field, which is the pulse height divided by sample thickness, carriers are transported to the counter electrode. Using a properly chosen pickup resistance, we measured the current transient with a Tektronix TDS754D oscilloscope and determined the carrier transit time from which the field-dependent mobility could be extracted.

\section{B. Experimental admittance curves}

We show in Fig. 1, the capacitance $C$ and conductance $G$ as function of the ac modulation frequency $f$ between $10^{2}$ and $10^{6} \mathrm{~Hz}$ and for applied de voltages $V_{0}$ from $3 \mathrm{~V}$ up to $10 \mathrm{~V}$. Above $3 \times 10^{5} \mathrm{~Hz}$, the $C-f$ curve for all voltages shows a plateau with an average value of $466 \mathrm{pF}$, followed by an abrupt decrease for a modulation frequency of about $4 \mathrm{MHz}$. The plateau is interpreted as the dielectric capacitance of the device. It is indeed consistent with the expected value obtained by taking a dielectric constant of 3 , a contact area $A$ of $0.235 \mathrm{~cm}^{2}$, and a layer thickness of $1340 \mathrm{~nm}$. The sharp cutoff which appears at high frequency (HF) leads further to a vanishing of the capacitance signal and is due to the effect of the series resistance $R_{S}$ of the whole structure. Among the multiple physical origins of the series resistance, contact resistance is one of the most probable ones. A minimum occurs in the intermediate-frequency (IF) region of the capacitance. For $V_{0}=3 \mathrm{~V}$, the position and magnitude of this minimum are, respectively, $7.1 \mathrm{kHz}$ and $389 \mathrm{pF}$. It is followed by a steplike transition leading to the HF plateau. When the applied dc voltage is increased, sensible variations of the $C$ - $f$ curve are observed. The low-frequency (LF) part of the curve decreases while, in the IF range, the amplitude of the extremum is enhanced and its position shifts to higher frequencies. The conductance curves show a constant value in the LF regime, followed by a general increase of the signal at high frequency. The height of the plateau increases with $V_{0}$, with a value of about $3.4 \times 10^{-5} \mathrm{~S}$ at $3 \mathrm{~V}$ and 2.1 $\times 10^{-4}$ at $10 \mathrm{~V}$. The region in which $G$ increases with $f$ starts at a threshold frequency depending on the applied bias and which is approximately equal to $2 \times 10^{5} \mathrm{~Hz}$ at the highest

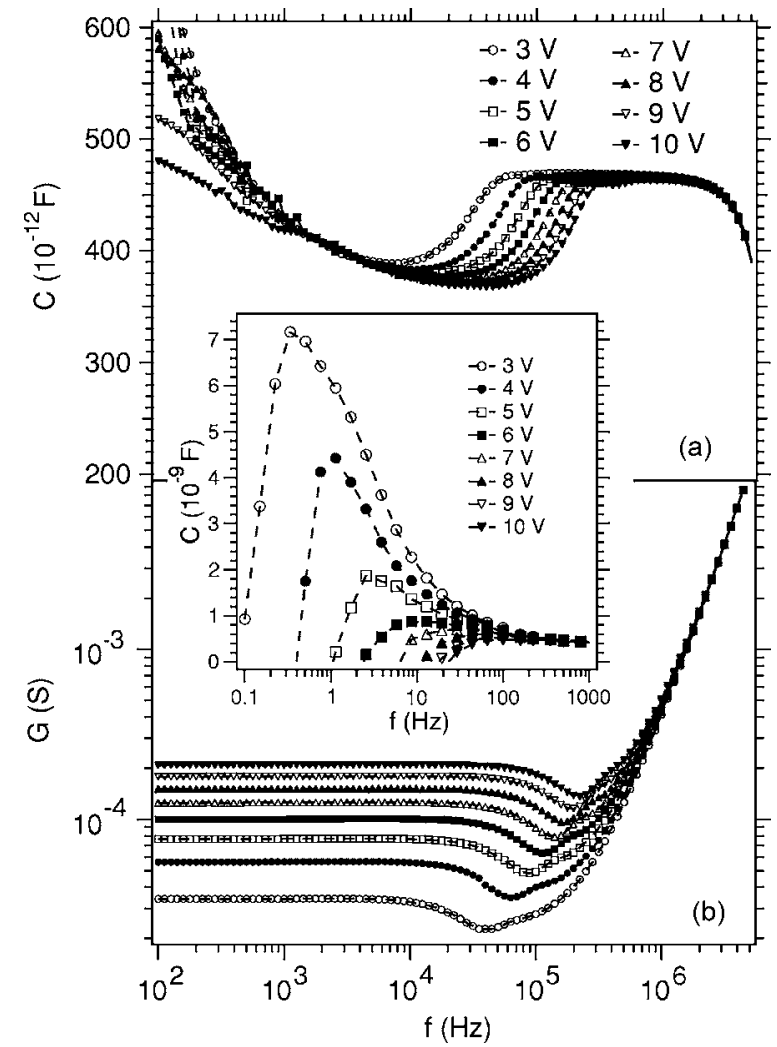

FIG. 1. Experimental (a) capacitance $C$ and (b) conductance $G$ as functions of the modulation frequency $f$ for applied dc voltages $V_{0}$ between $3 \mathrm{~V}$ and $10 \mathrm{~V}$ at room temperature. The inset shows the capacitance $C$ as a function of frequency $f$ in the low-frequency range $\left(0.1-10^{3} \mathrm{~Hz}\right)$ for the same applied dc voltage values.

voltage. This behavior at high frequency is related to the series resistance of the device. A small dip occurs in the IF region, just before the beginning of the increasing portion. Its position shifts into the HF region when the bias voltage increases.

The most interesting part of the admittance curves consists of the capacitance signal in the IF range-i.e., for $f$ roughly between $10^{3} \mathrm{~Hz}$ and $10^{5} \mathrm{~Hz}$. Indeed, it is believed that the average transit time $\tau_{\text {tr }}$ of the charge carriers can be evaluated from an interpretation of the negative differential susceptance function $-\Delta B=-\omega\left(C-C_{\mathrm{g}}\right)$, where $C_{\mathrm{g}}$ is the geometric capacitance of the organic layer and $\omega=2 \pi f$ the angular frequency of the ac oscillations. ${ }^{13,16}$ The position of the maximum in the $-\Delta B$ curve, $f_{\max }$, yields $\omega_{\max }=2 \pi f_{\max }$ which is equal to $\kappa \tau_{\text {tr }}^{-1}$, with $\kappa$ an empirical coefficient. In Fig. 2, we show the differential susceptance as function of the ac modulation frequency. The position and magnitude of the maximum in the $-\Delta B$ curve are dependent on the applied voltage $V_{0}$. When $V_{0}$ increases, the peak shifts to higher frequencies and its amplitude grows. One may formulate a remark at this stage: the method of determining carrier mobility by observation of the susceptance signal requires the condition that the total capacitance of the film must be lower than the dielectric value of $C_{\mathrm{g}}=\varepsilon A / d$, with $\varepsilon$ the permittivity of the organic layer, $A$ the contact area, and $d$ the layer thickness. The latter situation must occur at least for a nonnegligible frequency range. In the Section IV, we will show 


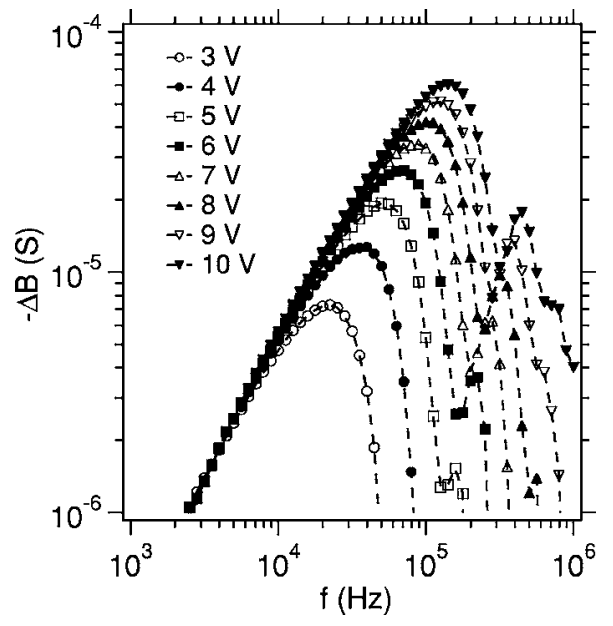

FIG. 2. Negative differential susceptance $-\Delta B$ curve as a function of frequency $f$ for applied dc voltages between $3 \mathrm{~V}$ and $10 \mathrm{~V}$.

that this is only one of the conditions required to evaluate mobility in organic materials by admittance spectroscopy.

The theoretical analysis, the background of which is developed hereafter, allows us to give an explanation, at the microscopic scale, of the existence of the minimum in the $C$ - $f$ curves. It will also provide a quantitative check of the whole procedure of obtaining mobility by admittance measurements. Determination of the value of the coefficient $\kappa$ can be performed by numerical calculations. As demonstrated in the discussion of Sec. IV, we obtained that $\kappa$ $\approx 3.2$, which is close to the values found by Blom et al. ${ }^{16}$ and Tsang et al. ${ }^{20}$ Using the peak position in the $-\Delta B$ curves at different applied voltages, we can subsequently evaluate the hole mobility, as illustrated in Fig. 3. The experimental points could be fitted by Poole-Frenkel's mobility fielddependence relation. We found a zero-field mobility $\mu_{h 0}$ of $(2.9 \pm 0.2) \times 10^{-4} \mathrm{~cm}^{2} /(\mathrm{V} \mathrm{s})$ and a characteristic electric field $E_{h 0}$ of $(1.52 \pm 0.28) \times 10^{5} \mathrm{~V} / \mathrm{cm}$. For comparison, hole mo-

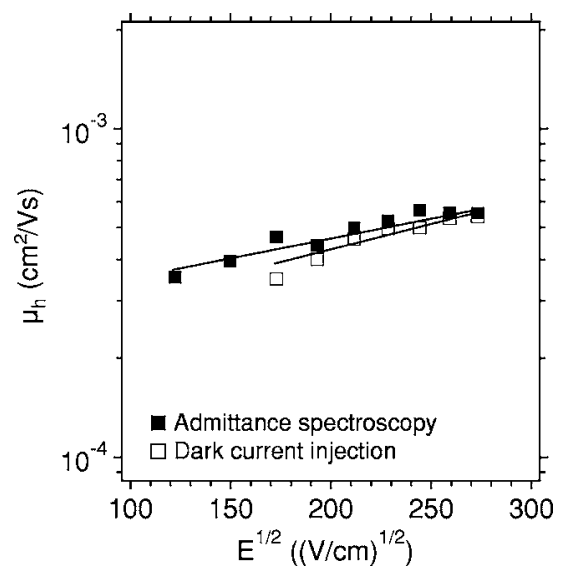

FIG. 3. Hole mobility $\mu_{h}$ in $\alpha$-NPD as a function of the square root of the applied electric field $E$. The experimental points (solid squares) were obtained by relating the carrier transit time to the peak in $-\Delta B(f)$ for different applied dc voltages. The fit is shown as the solid line. Experimental (open squares) and fit (solid line) results of dark current injection measurements. bility data obtained by dark current injection experiments are shown together in Fig. 3. Here, we found $\mu_{h 0}=(1.8 \pm 0.2)$ $\times 10^{-4} \mathrm{~cm}^{2} /(\mathrm{V} \mathrm{s})$ and $E_{h 0}=(5.4 \pm 0.7) \times 10^{4} \mathrm{~V} / \mathrm{cm}$. The values obtained by both techniques are comparable to those measured by Naka et al. using the time-of-flight technique. ${ }^{36}$

In addition to the IF region, the analysis of which allowed to determine hole mobility, admittance measurements at low frequency-i.e., $f<10^{2} \mathrm{~Hz}$ - show relevant information on electrical transport. In the inset of Fig. 1, a strong dependence of the capacitance on frequency and on applied bias can be observed. Negative values for $C$ are reported in a frequency range growing with the dc voltage. For $V_{0}=10 \mathrm{~V}$, starting with a value of $-3.7 \mu \mathrm{F}$ at $0.1 \mathrm{~Hz}$, the measured capacitance rapidly increases and reaches zero for $f$ between 20 and $30 \mathrm{~Hz}$. A general maximum occurs for each experimental curve, with a frequency position increasing with bias while the corresponding magnitude decreases. Negative capacitance shows that the $\mathrm{Al}$ electrode allows for a weak injection of electrons in the organic layer, which was not intentional. As developed in the following sections, the behavior of the system capacitance at very low frequency originates from the combination of the variations, with frequency, of the minority carrier concentration and the occupied trap concentration.

Our interpretation of the experimental results, based on a solution of the fundamental semiconductor equations for the structure consisting of a $\alpha$-NPD layer sandwiched by two electrodes, will show that the full description of the modulation of free carrier concentration, in magnitude and phase, can explain the capacitance response curves from which information on mobility is extracted. Furthermore, it demonstrates the role of the injected minority carriers on the LF behavior of the electrical quantities. The analysis is made by a detailed theoretical study of the small-signal electrical characteristics of the structure.

\section{THEORY}

We start from the basic equations for semiconductor devices $^{26}$ and extend these to organic compounds. The formal developments are applied to a structure with planar geometry containing one organic layer sandwiched between two metallic electrodes. Therefore, all quantities depend only on one spatial variable $x$. The treatments generally used to study devices made of inorganic substances are applied to the organic materials. ${ }^{37,38}$ The highest occupied molecular orbital (HOMO) levels play the role of the valence band and the lowest unoccupied molecular orbital (LUMO) levels give the conduction band. They are separated by an empty gap of value $E_{g}$. Holes are dominant in $\alpha$-NPD. The contribution of electrons, however, cannot be neglected in the transport properties for all frequency ranges. The importance of the role of the minority carriers is determined by the electron barrier height at the $\mathrm{Al}$ electrode. Assuming Boltzmann statistics, the free hole and electron concentrations are given in terms of the hole and electron quasi-Fermi levels $F_{p}$ and $F_{n}$ by

$$
p=N_{v} \exp \left[\left(E_{v}-F_{p}\right) / k T\right]
$$




$$
n=N_{c} \exp \left[\left(F_{n}-E_{c}\right) / k T\right],
$$

where $E_{v}$ and $E_{c}$ are the valence band edge and the conduction band edge while $N_{v}$ and $N_{c}$ are the effective valence band density of states and effective conduction band density of states, respectively. The band edges are position dependent and related to the electrostatic potential by

$$
\begin{gathered}
E_{c}=-\chi-q \psi, \\
E_{v}=-\chi-q \psi-E_{g},
\end{gathered}
$$

where $\chi$ is the electron affinity. It defines the position of the various energy levels with respect to a common zero reference level.

Defects are included with a density of states $D_{t}\left(E_{t}\right)$ depending on the defect energy $E_{t}$ inside the gap. ${ }^{37}$ The position dependence of $D_{t}$ could also be included. Integration of $D_{t}$ over the gap yields the total defect concentration $N_{t}$. The occupation function $f_{t}\left(E_{t}\right)$ is given at thermal equilibrium by Fermi-Dirac statistics. Defect states are either of donor or acceptor type. Acceptor states are neutral when empty and negatively charged when occupied by electrons. Donors are neutral when occupied and positively charged when empty. The number of occupied states, $n_{t}(x)$, at a given position $x$ is then obtained by integration, over the gap region, of the product $d_{t}\left(E_{t}\right)=f_{t}\left(E_{t}\right) D_{t}\left(E_{t}\right)$, where both factors can depend on position $x$.

The electrical potential $\psi$ obeys Poisson's equation

$$
\varepsilon \frac{d^{2}}{d x^{2}} \psi=-q\left(p-n+N_{D}-N_{A}+C_{t}\right),
$$

where $N_{D}$ and $N_{A}$ are the shallow donor and acceptor concentrations and $C_{t}$ is the trap charge concentration. The latter equals $-n_{t}$ for acceptor states and equals $\left(N_{t}-n_{t}\right)$ for donor states, $q$ being the electronic charge. The continuity equations for electrons, holes, and occupied defect states are

$$
\begin{gathered}
\frac{\partial p}{\partial t}=-\frac{1}{q} \frac{d}{d x} J_{p}-R_{p t}-R_{b b}, \\
\frac{\partial n}{\partial t}=\frac{1}{q} \frac{d}{d x} J_{n}-R_{n t}-R_{b b}, \\
\frac{\partial n_{t}}{\partial t}=R_{n t}-R_{p t} .
\end{gathered}
$$

In Eq. (6), $J_{p}$ is the hole current density consisting of a drift and a diffusion contribution, according to

$$
J_{p}=q p \mu_{h} E-q D_{h} \nabla p,
$$

where $E$ is the electric field, $\mu_{h}$ the hole mobility, and $D_{h}$ is the diffusion constant. A classical Einstein relation between diffusion constant and carrier mobility is assumed. Similarly, for electrons, we have

$$
J_{n}=q n \mu_{e} E-q D_{e} \nabla n .
$$

The band-to-band recombination term $R_{b b}$ is given by

$$
R_{b b}=B_{r}\left(n p-n_{i}^{2}\right),
$$

where $n_{i}$ is the intrinsic carrier density and $B_{r}$ a constant. The hole mobility is supposed to be field dependent, following a Poole-Frenkel law ${ }^{30}$

$$
\mu_{h}(E)=\mu_{h 0} \exp \left(\sqrt{\frac{E}{E_{h 0}}}\right) .
$$

Here $\mu_{h 0}$ is the zero-field mobility and $E_{h 0}$ is the characteristic electric field. The dependence of carrier mobility on charge density is neglected. A similar field dependence is also assumed for the electron mobility $\mu_{e}$.

The recombination term $R_{p t}$ is the total hole transition rate from trap states to the valence band. It results from the integration, over the band gap, of the hole transition rate per unit energy:

$$
r_{p t}\left(E_{t}\right)=c_{p} p f_{t} D_{t}-e_{p}\left(1-f_{t}\right) D_{t},
$$

where $c_{p}$ is the hole recombination constant and

$$
e_{p}=\left(\frac{c_{p}}{g}\right) N_{v} \exp \left[\frac{\left(E_{v}-E_{t}\right)}{k T}\right],
$$

with $g$ the degeneracy factor. Similarly, $R_{n t}$ is the integral over the band gap of the transition rate for electrons per unit energy:

$$
r_{n t}\left(E_{t}\right)=c_{n} n\left(1-f_{t}\right) D_{t}-e_{n} f_{t} D_{t},
$$

where $c_{n}$ is the electron recombination constant and

$$
e_{n}=g c_{n} N_{c} \exp \left[\frac{\left(E_{t}-E_{c}\right)}{k T}\right] \text {. }
$$

Each defect level is supposed to interact independently with the valence band and the conduction band. The interaction between trap states is neglected, which is certainly justified at trap concentrations for which hopping mechanisms between various defect states can be ignored.

Under steady-state conditions, Eq. (8) leads to the relation $r_{n t}\left(E_{t}\right)-r_{p t}\left(E_{t}\right)=0$, from which the steady-state occupation function can be deduced:

$$
f_{t 0}\left(x, E_{t}\right)=\frac{c_{n} n+e_{p}}{c_{n} n+e_{n}+c_{p} p+e_{p}} .
$$

This quantity is, in the general case, position dependent, as it depends on the hole and electron concentration. It reduces to the Fermi-Dirac shape only under thermal equilibrium conditions. This allows one to replace $f_{t}$ in the various expressions of $n_{t}$ and $R_{p t}$ in the steady-state equations by expression (17) and finally reduces the initial set of four equations to a set of three equations, as the defect variable is eliminated.

In the study of the ac response under small-signal conditions, a sinusoïdal potential of amplitude $\tilde{V}$ and of frequency $f=\omega / 2 \pi$ is added to the steady-state voltage $V_{0}$ :

$$
V=V_{0}+\tilde{V} e^{j \omega t}
$$

where $j=\sqrt{-1}$. Within the small-signal approximation, all quantities can be written as the sum of a steady-state plus a 
harmonic term. All higher-order terms are neglected. Labeling the steady-state term with an index 0 and the ac component with a tilde, one has, e.g., for the potential, the hole concentration, and the electron concentration,

$$
\begin{aligned}
& \psi(x, t)=\psi_{0}(t)+\widetilde{\psi} e^{j \omega t}, \\
& p(x, t)=p_{0}(t)+\widetilde{p} e^{j \omega t}, \\
& n(x, t)=n_{0}(t)+\widetilde{n} e^{j \omega t} .
\end{aligned}
$$

The amplitude of the various ac terms is complex in the general case, as these quantities are not in phase with the applied voltage. From the continuity equation for the defect states under the same assumption of noninteracting states, one can deduce the ac component of the occupation function. As for the steady-state case, the ac problem can be reduced to a set of three equations, obtained by introducing the harmonic developments (19)-(21) in the expression of Poisson's equation and the continuity equation for holes and electrons. The as-obtained equations are written in terms of the variables $\widetilde{\psi}, \widetilde{p}$, and $\widetilde{n}$. In addition to the hole and electron ac current densities $\widetilde{J}_{p}$ and $\widetilde{J}_{n}$, the total current density contains the displacement current term $\widetilde{J}_{D}=\varepsilon \partial E / \partial t$. The sum of these three terms yields the total current $\widetilde{J}$ which has to be constant with respect to position $x$. For the numerical resolution of both the steady-state and small-signal equations, one expresses all terms as functions of the electrical potential $\psi$ and of the hole and electron Fermi level $F_{p}$ and $F_{n}$, using relations (1) and (2) for the steady-state part. For the ac components of $p$ and $n$, one obtains by inserting expressions (20) and (21) into Eqs. (1) and (2), making use of Eqs. (4) and (3), and retaining only first-order terms

$$
\begin{gathered}
\tilde{p}=-p_{0}\left(q \widetilde{\psi}+\widetilde{F}_{p}\right) / k T, \\
\widetilde{n}=n_{0}\left(q \widetilde{\psi}+\widetilde{F}_{n}\right) / k T .
\end{gathered}
$$

The position-dependent occupied ac-defect-state concentration $\widetilde{n}_{t}(x)$ is obtained by numerically integrating the product of the defect density of states $D_{t}\left(E_{t}\right)$ and the ac-occupation function $\tilde{f}_{t}\left(x, E_{t}\right)$ :

$$
\tilde{n}_{t}(x)=\int_{E_{v}}^{E_{c}} \tilde{f}_{t}\left(x, E_{t}\right) D_{t}\left(E_{t}\right) d E_{t},
$$

where the integration is performed for energy values in the band gap. The steady-state and small-signal equations are of similar form; they therefore can be solved along the same numerical procedure. After scaling and discretization according to a variable-size mesh, the resulting second-order nonlinear equations are linearized in terms of small corrections of the variables and iterated until convergence is achieved.

Contacts are supposed to be of the Schottky type, where hole and electron currents are specified at the left contact $x_{L}$ and the right contact $x_{R}$. In the expression of the boundary conditions, each contact current consists of a thermionic and recombination current $J_{1}$ plus a tunnelling term $J_{2} \cdot{ }^{25}$ The first term $J_{1}$ can be expressed in terms of a surface recombination velocity and is written, respectively, for holes and electrons ${ }^{39}$

$$
\begin{aligned}
& J_{1 p}=q v_{s p}\left(p-p_{\mathrm{eq}}\right), \\
& J_{1 n}=q v_{s n}\left(n-n_{\mathrm{eq}}\right),
\end{aligned}
$$

where $p_{\mathrm{eq}}$ and $n_{\mathrm{eq}}$ are, respectively, the hole and electron thermal equilibrium values at the contacts while $v_{s p}$ and $v_{s n}$ are the surface recombination velocities for holes and electrons. The electron and hole current densities $J_{2}$ at the contacts are the Fowler-Nordheim tunneling currents. The electron term is written

$$
J_{2 n}=\alpha_{t}\left(\frac{B}{\phi_{n}}\right) E^{2} \exp \left[-\alpha_{b} C\left(\frac{\left(q \phi_{n}\right)^{3 / 2}}{E}\right)\right],
$$

where $\phi_{n}$ is the electron barrier height, $B=q^{3} /(8 \pi h)$, and $C=2 \pi \sqrt{2 m} /(3 h q), h$ being Planck's constant. The quantities $\alpha_{t}$ and $\alpha_{b}$ are adjustable parameters allowing for eventual corrections to take into account for the inadequate field dependence of these expressions. Image force lowering is also included.

This yields all $\mathrm{dc}$ and ac components of the involved physical quantities. The small-signal conductance $G$ and capacitance $C$ are obtained from the complex admittance $Y=\widetilde{J} / \tilde{V}$ which is decomposed into an equivalent parallel conductance and capacitance:

$$
Y=G\left(\omega, V_{0}\right)+j \omega C\left(\omega, V_{0}\right) .
$$

These quantities can be compared with experimentally obtained admittance data.

\section{ANALYSIS AND DISCUSSION}

\section{A. Microscopic description of the device}

In order to completely describe the system, one needs the parameters corresponding to the semiconducting material, the electrodes, and the external conditions such as dc bias or modulation frequency. The structure on which the measurements have been performed corresponds to a pair of Schottky contacts connected back to back with the $\alpha$-NPD layer as a conducting channel, the thickness of which is $d=1340 \mathrm{~nm}$. For the numerical calculations, we have taken $\left(E_{\mathrm{vac}}-E_{v}\right)$ $=5.5 \mathrm{eV}$ and $\left(E_{c}-E_{v}\right)=3.1 \mathrm{eV}$, with the vacuum level $E_{\text {vac }}$ being the energy reference. The ITO/ $\alpha$-NPD interface shows a nearly Ohmic contact behavior with a barrier height for holes $q \phi_{p}$ of $0.2 \mathrm{eV}$. For the barrier height for electrons $q \phi_{n}$ at the $\mathrm{Al}$ cathode, we have studied two different cases. The first one, in which a value of $1.6 \mathrm{eV}$ holds for $q \phi_{n}$, leads to a system where only holes, injected at the ITO electrode, play a role in the transport properties of the device. In the second case, with a value of $0.4 \mathrm{eV}$ for the $\mathrm{Al}$ barrier, electrons are injected into the organic layer and contribute to the electrical characteristics. A theoretical built-in voltage $V_{\text {bi }}$ $=1.3 \mathrm{eV}$ can be deduced for the first case while a value of $2.5 \mathrm{eV}$ should be assumed for the second case. As demonstrated in the following, the small-signal characteristics of the real device at low frequency can be numerically reproduced by the introduction of trap levels in the band gap of 


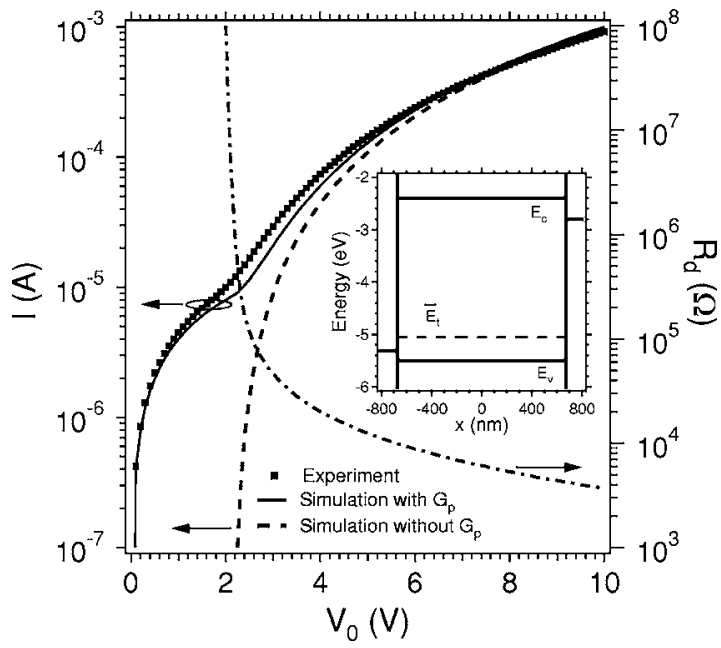

FIG. 4. Experimental (solid squares) and theoretical (lines) current-voltage characteristics of the ITO/ $\alpha$-NPD/Al system. The result including the effect of parallel conductance $G_{p}$ is shown as solid lines, with $G_{p}=4 \times 10^{-6} \mathrm{~S}$. $R_{d}$ is the differential resistance. The inset shows the flat energy band diagram of the system.

the organic material. Traps are included with a Gaussian distribution of states centered at energy $\bar{E}_{t}=0.45 \mathrm{eV}$ and of width $\sigma_{t}=0.4 \mathrm{eV}$. In the numerical simulation, their effect can be switched on and off by setting the corresponding concentration to the adequate level in the input data.

The basic semiconductor equations are first solved under steady-state conditions. The values of the space-dependent carrier concentrations are determined by Eqs. (1) and (2) where the quasi-Fermi levels $F_{p}$ and $F_{n}$ for holes and electrons, respectively, at zero voltage coincide with the Fermi level $E_{F}$ of the system in thermal equilibrium. For any given applied voltage, the electrostatic potential and the steadystate concentrations of holes, electrons, and occupied traps are thus obtained. Using Eqs. (9) and (10), the carrier current densities are calculated and the total current $I$ deduced. In Fig. 4, we show the theoretical current-voltage characteristic and the numerically obtained differential resistance of the device $R_{d}=(d I / d V)^{-1}$. A good agreement with the experiment is observed when an additional Ohmic term is added to the total current of the device. This contribution is only dominant for voltages below $2 \mathrm{~V}$ and simulates the effect of leakage currents. In the equivalent circuit representation of the system, it takes the form of a parallel conductance $G_{p}$ $=4 \times 10^{-6} \mathrm{~S}$. We may thus clearly identify two distinct regimes in the steady-state characteristic. The main one starts from $V_{0} \approx 2 \mathrm{~V}$ and increases with a rate weaker than that for an exponential law, monotonically reducing with the bias. Values as high as $10^{-3} \mathrm{~A}$ are measured at $10 \mathrm{~V}$. The simulation is performed with an electron barrier height of $0.4 \mathrm{eV}$. The flat energy band diagram of the corresponding system is given for reference as the inset of Fig. 4.

Once obtained, the steady-state solution serves as a basis for the calculation of the small-signal components, according to Eqs. (22) and (23). As an example, we show in Fig. 5 the real parts of the ac concentrations of holes, $\widetilde{p}^{R}$, electrons, $\widetilde{n}^{R}$, and occupied trap states, $\widetilde{n}_{t}^{R}$, as functions of the space coordinate $x$, for $V_{0}=5 \mathrm{~V}$ and modulation frequency of $0.1 \mathrm{~Hz}$.

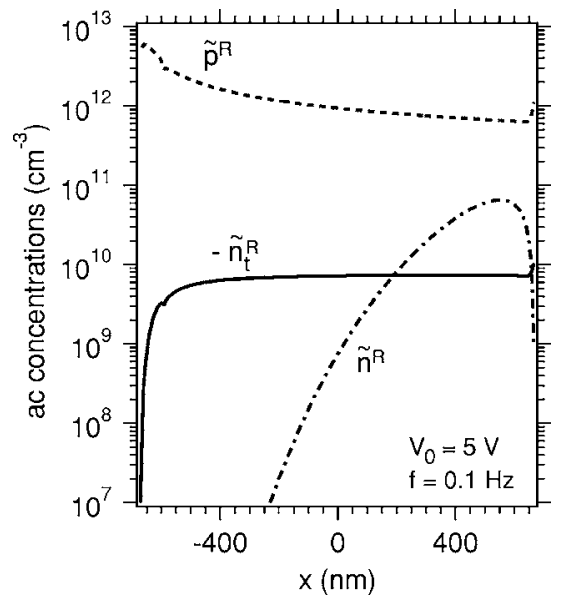

FIG. 5. Real parts of the small-signal ac hole concentration $\tilde{p}$, electron concentration $\tilde{n}$, and occupied trap concentration $\tilde{n}_{t}$ as functions of the $x$ coordinate for applied dc voltage $V_{0}=5 \mathrm{~V}$ and modulation frequency of $0.1 \mathrm{~Hz}$. The electron barrier height $q \phi_{n}$ is taken as $0.4 \mathrm{eV}$.

These quantities are calculated using the set of parameters given in Table I. The hole concentration is the dominant contribution throughout the device, with a background value of about $10^{12} \mathrm{~cm}^{-3}$ in the bulk and a maximum very close to the ITO contact. As expected, the electron modulation is important only in the cathode region, presenting an extremum at $15 \mathrm{~nm}$ from the contact. Outside the peak, Re $\widetilde{n}$ rapidly decreases to negligible concentrations. At low frequency, the ac concentration of occupied trap states is nearly constant in the organic layer, only showing strong variations near the anode. In the bulk, an average magnitude of $6 \times 10^{9} \mathrm{~cm}^{-3}$ is reported.

TABLE I. Parameters of the numerical simulation.

\begin{tabular}{lc}
\hline \hline Parameter & Value \\
\hline Thickness $d$ & $1340 \mathrm{~nm}$ \\
Contact area $A$ & $0.235 \mathrm{~cm}^{2}$ \\
Dielectric constant $\varepsilon_{r}$ & 3 \\
Parallel conductance $G_{p}$ & $4 \times 10^{-6} \mathrm{~S}$ \\
Series resistance $R_{s}$ & $45 \Omega$ \\
Band gap $E_{g}$ & $3.1 \mathrm{eV}$ \\
Hole zero-field mobility $\mu_{h 0}$ & $3 \times 10^{-4} \mathrm{~cm}^{2} / \mathrm{V} \mathrm{s}$ \\
Hole characteristic field $E_{h 0}$ & $1.74 \times 10^{5} \mathrm{~V} / \mathrm{cm}$ \\
Electron zero-field mobility $\mu_{e 0}$ & $1 \times 10^{-9} \mathrm{~cm}^{2} / \mathrm{V} \mathrm{s}$ \\
Electron characteristic field $E_{e 0}$ & $1.74 \times 10^{5} \mathrm{~V} / \mathrm{cm}$ \\
Valence band effective density of states $N_{v}$ & $1 \times 10^{21} \mathrm{~cm}^{-3}$ \\
Conduction band effective density of states $N_{c}$ & $1 \times 10^{21} \mathrm{~cm}^{-3}$ \\
Band-to-band recombination coefficient $B_{r}$ & $2 \times 10^{-14} \mathrm{~cm}^{3} / \mathrm{s}$ \\
\hline Hole capture constant $c_{p}$ & $4 \times 10^{-13} \mathrm{~cm}^{3} / \mathrm{s}$ \\
Electron capture constant $c_{n}$ & $1 \times 10^{-18} \mathrm{~cm}^{3} / \mathrm{s}$ \\
Trap total concentration $N_{t}$ & $8 \times 10^{13} \mathrm{~cm}^{-3}$ \\
Mean energy of traps $\left(E_{c}-\bar{E}_{t}\right)$ & $2.65 \mathrm{eV}$ \\
Width of the trap Gaussian distribution $\sigma_{t}$ & $0.4 \mathrm{eV}$ \\
\hline \hline
\end{tabular}




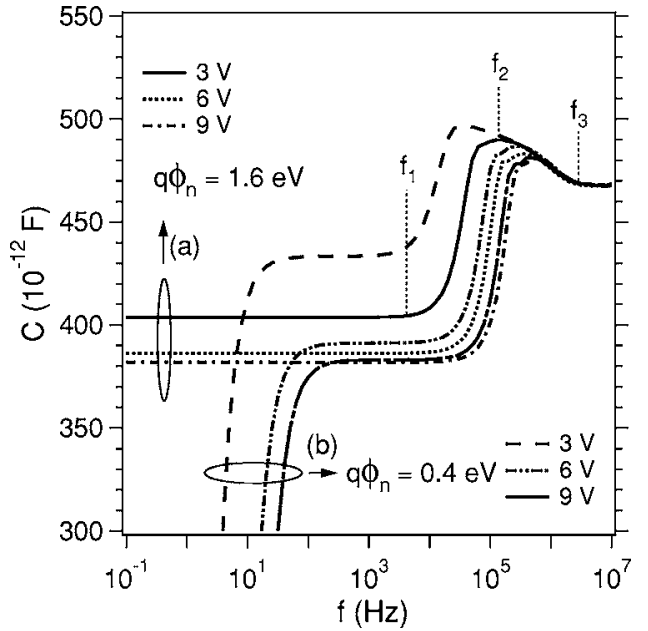

FIG. 6. Capacitance $C$ as a function of frequency $f$ for applied voltage $V_{0}=3,6$, and $9 \mathrm{~V}$. Set (a) is related to the case where the barrier height at the right contact (Al) $q \phi_{n}$ is of $1.6 \mathrm{eV}$ while set (b) corresponds to the situation where $q \phi_{n}=0.4 \mathrm{eV}$. Series resistance $R_{S}$ is set to zero.

\section{B. Admittance response of the ideal trap-free system}

We first discuss the small-signal response of the structure without traps. This step allows us to explain the role of the majority carriers, as well as that of the minority carriers, in the frequency-dependent complex admittance and impedance curves. By setting aside the effect of trapped charges, the physical mechanisms at the basis of the transport of free charges can be clarified. In the following, the contact area will be fixed by normalization of the high-frequency value of the capacitance to the corresponding experimental value, leading to $A=0.235 \mathrm{~cm}^{2}$. In order to illustrate the role of hole modulation in the admittance response curves, the electrical characteristics are calculated at various applied voltages for a trap-free device. In this application, the dielectric constant $\varepsilon_{r}$ is taken as 3. Figure 6 gives the resulting $C-f$ curves between $10^{-1}$ and $10^{7} \mathrm{~Hz}$. In order to fully understand the role of the modulation of majority carrier concentration, the injection of electrons from the cathode will be neglected in a first step. Its effect will be discussed in a next step. In the simulation leading to the results shown in Fig. 6, series resistance effects are neglected. Therefore, we do not observe any cutoff frequency in the HF region of the capacitance. Instead, the HF value of $C$ corresponds to the geometrical or dielectric capacitance $C_{\mathrm{g}}$ defined above. The transit time effect clearly appears on the admittance curves. Indeed, for frequencies below $10^{4} \mathrm{~Hz}$, the value of $C$ is smaller than that of the geometrical limit. This corresponds to the "inductive" contribution as described in the literature. ${ }^{15,19}$ For each value of $V_{0}$, the capacitance remains constant up to a frequency value $f_{1}$, above which $C$ increases up to a frequency $f_{2}$. It then decreases down to the value of $C_{\mathrm{g}}$ at a frequency $f_{3}$. As an example, for $V_{0}=3 \mathrm{~V}$, one has $f_{1}=4.0 \times 10^{3} \mathrm{~Hz}, f_{2}=1.3$ $\times 10^{5} \mathrm{~Hz}$, and $f_{3}=3.0 \times 10^{6} \mathrm{~Hz}$. The values of $f_{1}$ and $f_{2}$ increase with $V_{0}$. An increasing value of the applied voltage implies a reduction of the transit time and, as a consequence, an increasing value of the transition frequencies. This leads to a HF shift of the capacitance step defined by the $f_{1}-f_{2}$ region. Hole injection into the organic film starts as soon as the built-in potential is exceeded. In the semiconducting material, the building up of the electric field is related to the presence of the accumulation of the space-charge density. For $V_{0} \geqslant 2 \mathrm{~V}$, the superposition of the small-signal alternating field leads to modulation of the space charge, along time and $x$ coordinate, in order to adapt to the variations induced by the oscillations. An inductive behavior of the capacitance is observed due to this charge redistribution when the period $T=1 / f$ of the ac voltage is longer than the carrier transit time $\tau_{\text {tr }}$, which represents the duration of a travel from one electrode to the other one. The related negative variation of the capacitance, with respect to the dielectric value, originates from the phase lag between the applied stimulus and the subsequent relaxation of the space charge. When the frequency is much higher than $1 / \tau_{\text {tr }}$, the capacitance meets the geometrical value. At the microscopic level, the small-signal concentration of holes, as represented in Fig. 5, vanishes at high frequencies. The interpretation of the admittance spectra in the light of the analysis of microscopic quantities such as the ac concentration allows us to explain the origin of the transit time effect. The latter is therefore due to a modulation of the hole charge inside the structure. Above a frequency which is of the order of the reciprocal transit time, the holes no longer respond to the modulating applied voltage and the capacitance is that of a classical dielectric.

Although the concentration of electrons is much lower than the concentration of holes in $\alpha$-NPD, the contribution of the minority carriers to the total capacitance of the system cannot be neglected when the barrier height at the cathode is sufficiently small to allow for electron injection. As illustrated in Fig. 6, the enhanced presence of electrons in the device strongly modifies the admittance spectra. In the IF region, the capacitance response increases in magnitude for all dc voltages while the position of the transit time step is slightly shifted to lower frequencies. The negative variation of $C$ between $3 \times 10^{3} \mathrm{~Hz}$ and $2 \times 10^{5} \mathrm{~Hz}$, however, holds, which guarantees that hole mobility can still be deduced from the modified curves. The most important changes are in the LF region, where the capacitance signal abruptly vanishes and shows negative values as low as $-6.3 \times 10^{-8} \mathrm{~F}$ for $V_{0}=9 \mathrm{~V}$ at $0.1 \mathrm{~Hz}$. The frequency range for which $C$ is negative widens when the bias increases. These features can be interpreted by putting forward arguments similar to those invoked to explain the reduction of the capacitance at frequencies below the reciprocal transit time. ${ }^{21}$ Indeed, the space charge present in the organic layer leads to a deviation of the capacitance $C$ from its dielectric value measured at high frequency. Because of the lag of the space charge increasing with respect to the applied ac voltage, the variation of $C$ is negative. When electrons are injected in the devicei.e., bipolar conduction occurs - the additional space charge can be much larger than in the unipolar case, due to the mutual compensation of both free carrier types. ${ }^{34}$ As a consequence, the capacitance deviation from the geometrical limit increases in magnitude, leading to negative values for the total capacitance. The amplitude of the negative capacitance effect of minority carriers depends on the value of the recombination constant $B_{r} \cdot{ }^{19}$ For the results shown in Fig. 6, 
we have chosen $B_{r}=2 \times 10^{-14} \mathrm{~cm}^{3} / \mathrm{s}$. It can be related to relatively high recombination rates as the frequency region where hole transit mechanism takes place is not much modified by the electron injection. This result shows that, when supported by numerical simulation, the admittance spectroscopy technique, performed for a wide frequency range and under various applied voltages, can provide a way to estimate the strength of carrier recombination in organic materials.

\section{Admittance curves with a Gaussian distribution of defect states}

The oscillation of the hole concentration near the injection electrode is responsible for the negative capacitance contribution at intermediate frequencies. It cannot, however, explain the LF behavior of $C$. The so-called excess capacitance at low frequency was either reproduced by a frequencydependent mobility ${ }^{15,17,20}$ or by a dispersion of the transit time values. ${ }^{15}$ Another explanation is that one or several trap states can be partially responsible for the variation of $C$ for $f$ smaller than the transit frequency. ${ }^{18}$ In this paper, we show that a Gaussian distribution of trap states, located near the valence band edge, can account for this behavior of the capacitance. Here again, the analysis of the microscopic quantities allows us to give an interpretation of the frequencydependent complex admittance. In our approach, the trap states are explicitly included in the numerical simulation, as indicated by Eq. (8).

The mean energy of the donor-type traps $\bar{E}_{t}$ is located at $0.45 \mathrm{eV}$ from the valence band edge. This position is close to the Fermi level. This ensures that a maximum effect occurs when the applied voltage is modulated. It is indeed well known that the contribution of traps to the device capacitance is maximum for those levels which cross the Fermi level. ${ }^{40}$ This also allows us to reproduce the main effects of a continuous distribution of trap states, as levels well above the Fermi energy do not contribute, as they remain empty, and those well below the Fermi level do not contribute either, as they remain fully occupied. We have taken a distribution width of $\sigma_{t}=0.4 \mathrm{eV}$ and a total trap concentration $N_{t}$ of 8 $\times 10^{13} \mathrm{~cm}^{-3}$. The result for the detailed response of the individual trap states at dc bias $V_{0}=5 \mathrm{~V}$ and modulation frequency $f=1 \mathrm{~Hz}$ is shown in Fig. 7 for the particular case $x$ $=0 \mathrm{~nm}$. This position is representative of the situation in the bulk, where the trap response is known to be practically constant with respect to the space coordinate, as shown in Fig. 5. On the one hand, together with the density of states $D_{t}$, the steady-state occupied-state concentration $d_{t 0}=D_{t} f_{t 0}$ is given as a function of trap energy $E_{t}$. The shape of $d_{t 0}$ depends on the local position of the quasi-Fermi level, which varies with the external dc voltage. For applied bias of $5 \mathrm{~V}$, the occupation rate of trap states in the bulk is smaller than half of the total concentration while the contribution of traps to the electrostatic charge is determined by the crossing of the hole quasi-Fermi level with the defect band. On the other hand, we observe that the real part of the ac occupation function is the dominant component at this frequency. It describes the filling and emptying of the trap energies $E_{t}$ between 0.25 and

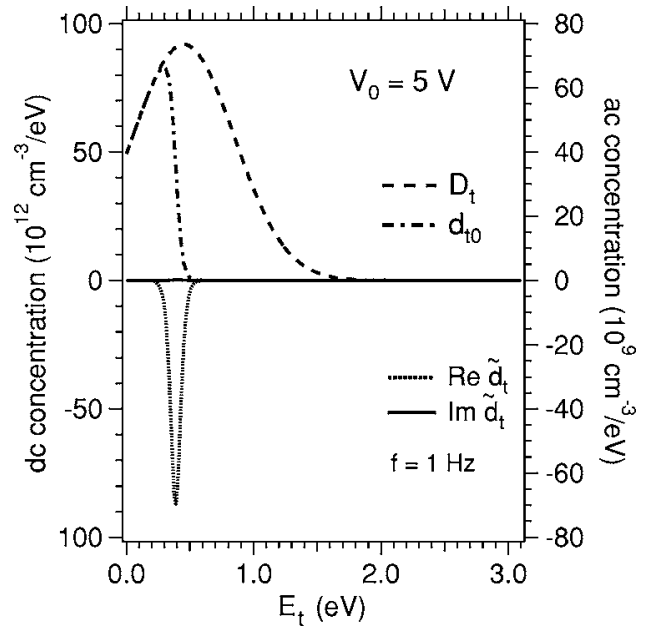

FIG. 7. Density of trap states $D_{t}$, steady-state occupied trap concentration $d_{t 0}$, real part, and imaginary part of the small-signal occupied trap concentration, $\operatorname{Re} \tilde{d}_{t}$ and $\operatorname{Im} \tilde{d}_{t}$, as functions of the trap energy $E_{t}$, for $x=0$. The energy reference of $E_{t}$ is here the valence band edge. The small-signal quantities are shown for frequency $f=1 \mathrm{~Hz}$.

$0.50 \mathrm{eV}$ above the valence band edge. At $x=0 \mathrm{~nm}$, the response is only due to the states whose position is close to that of the hole quasi-Fermi level, the other states remaining either occupied or empty. Our calculations also show that the magnitude of the trap response remains strong up to a frequency of about $10 \mathrm{~Hz}$, which is much lower than the value of $f_{1}$ at moderate voltage. For $f>10^{2} \mathrm{~Hz}$, the role played by the defect band vanishes. This frequency-dependent behavior originates from the limited ability of traps to adapt to the applied ac voltage and can be illustrated by the definition of a defect transition frequency $f_{d}$ which value ranges between $10 \mathrm{~Hz}$ and $10^{2} \mathrm{~Hz}$. For frequencies above $f_{d}$, defect states cannot follow the small-signal modulation anymore, involving the extinction of the trap contribution.

In the following step, the capacitance of the system containing traps can be obtained. In Fig. 8, the $C$ - $f$ curves are shown for three different bias values. When electron injection is negligibly small, the effect of the trap levels is to give an additional contribution to the capacitance at low frequencies. The amplitude of the contribution, defined here as the difference between the LF plateau and the capacitance due to the transit time effect, increases with the applied dc voltage. The trap cutoff frequency, which is between $25 \mathrm{~Hz}$ and $40 \mathrm{~Hz}$, does not strongly depend on the dc voltage. Its value is mainly determined by the trap parameters. At $3 \mathrm{~V}$, the resulting $C$ - $f$ curve presents a minimum in the region $10^{2}-10^{4} \mathrm{~Hz}$. The presence of the trap levels leads to the filling of the negative contribution originating from the transit time effect. From the curves of Fig. 8, one can see that, in order to preserve a region where $C$ is lower than the geometrical value, the trap contribution must not be too important, as the valley in the $C-f$ curve would then be completely filled and no mobility evaluation would be possible. The ac curves strongly depend on the parameters used to describe the trap states. Yet for organic compounds, their precise value is not known in any case. As shown by Eqs. (13) and 


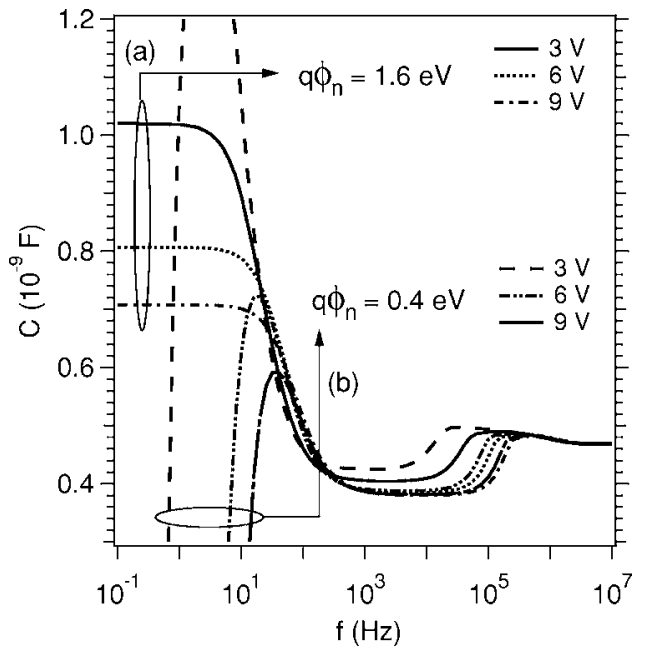

FIG. 8. Capacitance $C$ as a function of frequency $f$ for applied voltage $V_{0}=3,6$, and $9 \mathrm{~V}$. Set (a) is related to the case where the barrier height at the right contact (Al) $q \phi_{n}$ is of $1.6 \mathrm{eV}$ while set (b) corresponds to the situation where $q \phi_{n}=0.4 \mathrm{eV}$. Traps are present with a concentration of $8 \times 10^{13} \mathrm{~cm}^{-3}$ and series resistance $R_{S}$ is set to zero.

(14), the trap response essentially depends on the energy position $E_{t}$ relative to the valence band and the capture coefficient $c_{p}$. The total concentration of traps $N_{t}$ is also an important parameter of the simulation, as it has an effect on the frequency dependence of the capacitance in the LF region.

When electrons are allowed to flow into the organic device, by a reduced barrier height at the $\mathrm{Al}$ cathode, the $\mathrm{LF}$ region of the admittance curves is again deeply modified. The plateau corresponding to the trap contribution disappears at all voltages, and the value of $C$ vanishes and becomes negative. At $V_{0}=3 \mathrm{~V}$, a local maximum occurs at a frequency of $2.5 \mathrm{~Hz}$, with a magnitude of $1.5 \mathrm{nF}$. In the IF region, the transit time step is shifted to lower frequency. Minority carrier injection has therefore an effect on the values of the majority carrier mobility determined by the position of this transition, with or without traps in the system. When traps are present and minority carrier injection occurs, the obtained curves result from a combination of the capacitance responses of set (b) of Fig. 6 and set (a) of Fig. 8.

The last step of the analysis is to reproduce the experimental curves obtained for the ITO/ $\alpha$-NPD/Al system. To achieve this, we performed a numerical simulation of the capacitance and the conductance over a wide range of the above-mentioned microscopic parameters. Our goal is to obtain the correct transit frequencies, negative capacitance deviations with respect to the geometrical limit, defect transition frequencies, and low-frequency capacitance amplitudes. These are indeed the most salient features of the measured electrical characteristics. Perfect agreement with the experiment cannot be expected for such a complex system. Implicit approximations such as homogeneity or abrupt barriers are not necessarily valid in a real device. The results are shown in Fig. 9 for applied de voltage varying from $3 \mathrm{~V}$ to $10 \mathrm{~V}$. The most essential values of the obtained parameters are $\left(\bar{E}_{t}-E_{v}\right)=0.45 \mathrm{eV}, \sigma_{t}=0.95 \mathrm{eV}$, and $N_{t}=8 \times 10^{13} \mathrm{~cm}^{-3}$. The

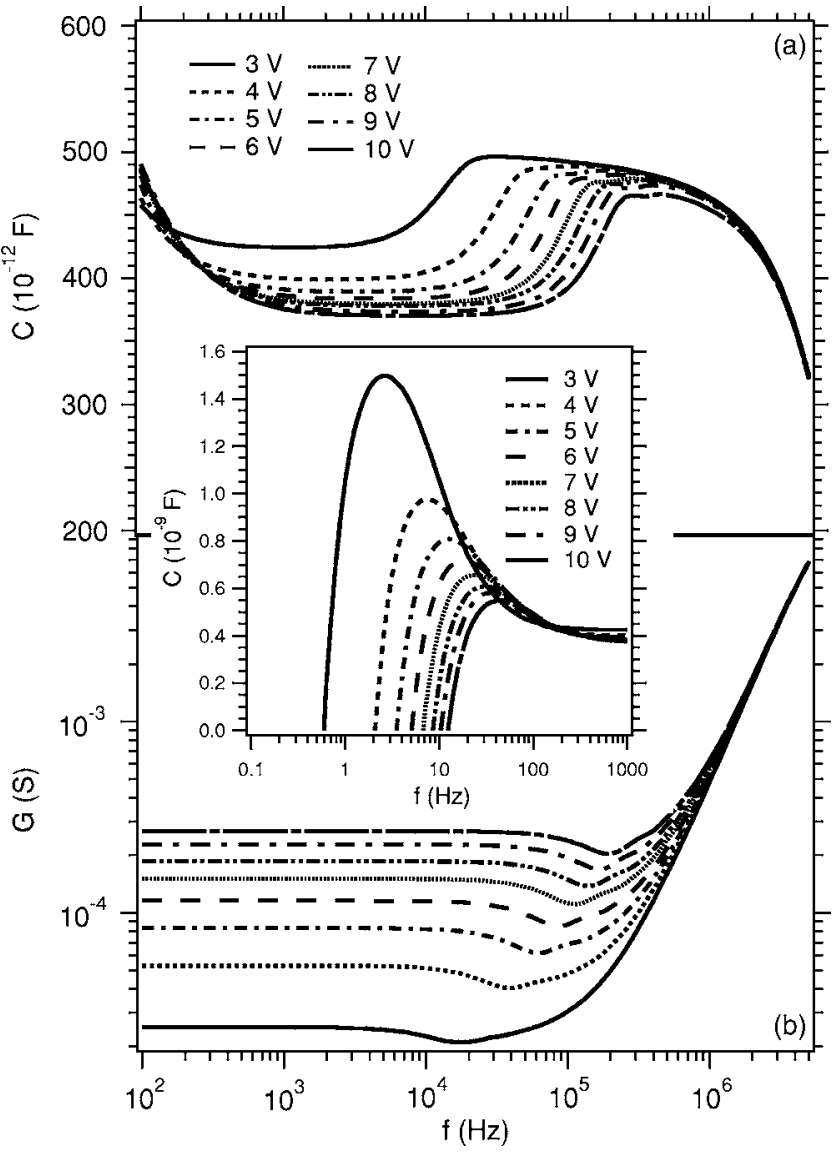

FIG. 9. Theoretical (a) capacitance $C$ and (b) conductance as functions of the frequency $f$ for dc bias $V_{0}$ between $3 \mathrm{~V}$ and $10 \mathrm{~V}$. Parameters of the simulation are of Table I, with $R_{s}=45 \Omega$ and $G_{p}=4 \times 10^{-6} \mathrm{~S}$. The inset shows the capacitance $C$ as function of frequency $f$ in the low-frequency range $\left(0.1-10^{3} \mathrm{~Hz}\right)$ for the same applied dc voltage values.

full set of parameters is given in Table I. The theoretical voltage-dependent $C-f$ and $G-f$ curves well reproduce the experimental results shown in Fig. 1. The series resistance effect was included in the numerically obtained capacitance and conductance. Furthermore, in order to correctly simulate the low-frequency values of the conductance, we have added the real term $G_{p}$ to the complex admittance $Y$ of the structure. This consequently allows us to reproduce the shape of the steady-state current-voltage characteristic. The variation of the LF region of the calculated capacitance curves with applied bias, as well as the amplitude of the minimum and the shift of the carrier transit-induced frequency, reproduced the characteristics of the experimental data. The admittance spectra could be refined by introducing several Gaussian distributions of traps in the input data. That would result in less abrupt defect transitions and smoother capacitance variations at low frequency.

Theoretical studies by numerical simulation allow us to formulate the conditions which have to be fulfilled in order to evaluate carrier mobility by the technique of admittance spectroscopy. First, there must be an inductive contribution to the capacitance from the transit time effect. This is achieved by carrier injection at high level, which is allowed 
by an Ohmic behavior of the injecting electrode-i.e., very low barrier height at the contact. In order to be correctly measured, the reciprocal transit time should be included in the frequency range of the impedance measurement tool. For a given analyzer, it is then possible to define a range of mobility values that can be extracted by the method. Second, the trap concentration has to be sufficiently low in order to maintain a minimum in the $C-f$ curve. Third, the trap cutoff frequency should be sufficiently low, at least lower than the transit frequency. One could estimate that a difference of about two decades between the carrier transit frequency and the defect transition frequency is necessary to yield an observable maximum in the negative differential susceptance curve. In most cases, however, these two latter conditions can hardly be efficiently controlled. Finally, one last condition is that, when both carrier types are active, their expected mobilities are different by several orders of magnitudes. Otherwise, the capacitance variation corresponding to the modulation of the concentration of one carrier type would interfere with that of the other carrier type. Such a complex situation would ruin the practical utility of admittance spectroscopy as a mobility measurement tool. In $\alpha$-NPD, as holes are clearly dominant over electrons for transport properties, frequencydependent experiments lead to satisfactory results. The electron mobility $\mu_{e}$ could not be evaluated. The corresponding peaks in the negative differential susceptance, which would appear for frequencies below $10 \mathrm{~Hz}$, are concealed by trap response and negative capacitance effect. The inset of Fig. 9 shows the calculated capacitance spectra between $0.1 \mathrm{~Hz}$ and $1 \mathrm{kHz}$ for voltages up to $10 \mathrm{~V}$. It reproduces the characteristic features of the experimental data shown in the inset of Fig. 1 -i.e., negative values of $C$ at very low frequency and a local peak due to the combined effect of recombination and traps.

Finally, we would like to comment on the results obtained for the hole mobility in $\alpha$-NPD. The numerically obtained differential susceptance curves are given in Fig. 10. The voltage dependence of the peak positions and amplitudes reproduces well the experimental data of Fig. 2. For each value of $V_{0}$, we obtain the majority carrier transit time $\tau_{\text {tr }}$ by taking the position of the corresponding maximum $f_{\max }$. The coefficient $\kappa$ between $\omega_{\max }$ and $\tau_{\text {tr }}^{-1}$ was determined by a linear fit of the relation between these two latter quantities. The values of $\omega_{\max }=2 \pi f_{\max }$ were extracted from the $-\Delta B(f)$ curves at different dc voltages while those of $\tau_{\text {tr }}$ were obtained making use of Poole-Frenkel's field-dependent mobility introduced as input in the simulation and $E=\langle E\rangle=\left(V_{0}-V_{\mathrm{bi}}\right) / d$, the mean value of the electric field. The fit procedure yields a value of $\kappa=3.2 \pm 0.1$, which was subsequently used in the interpretation of the experimental admittance spectra. The same value then allows one to evaluate the carrier mobility from the theoretical susceptance curves. In Fig. 11, the resulting output is compared to the input data of the calculations. The purpose of this plot is first to demonstrate the selfconsistency of our approach, as the obtained values of the mobility can reproduce the field-dependence model introduced in the numerical code. Second, it shows that admittance spectroscopy experiments can be applied to determine carrier mobility in organic materials with an acceptable accuracy. The comparison with the result of Fig. 3 shows a

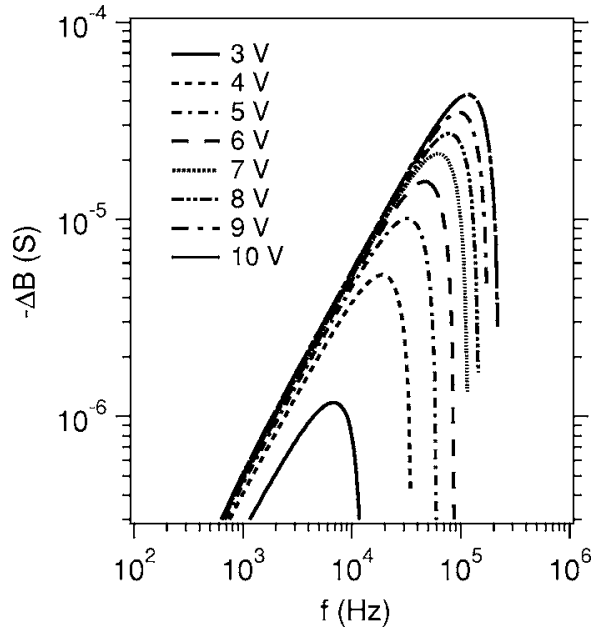

FIG. 10. Calculated negative differential susceptance $-\Delta B$ curve as a function of frequency $f$ for applied dc voltages between $3 \mathrm{~V}$ and $10 \mathrm{~V}$. Series resistance $R_{S}$ is equal to zero.

good agreement between the experimental and theoretical values of $\mu_{h}$. We notice, however, that for an electric field lower than $2 \times 10^{4} \mathrm{~V} / \mathrm{cm}$, the mobility obtained by the method is overestimated. The origin of this discrepancy is that, in the classical determination of $\mu_{h}$, one makes use of the averaged value of the electric field. Yet as demonstrated by the result shown in the inset of Fig. 11, the electric field is far from being rigorously constant throughout the organic layer. One should be aware that the method leads to a value of mobility averaged over the width of the organic layer. For larger bias values, we illustrate here that the field dependence of hole mobility in $\alpha$-NPD can be well reproduced by PooleFrenkel's law. Moreover, within our approach, the origin of the negative contribution to the capacitance, as well as the

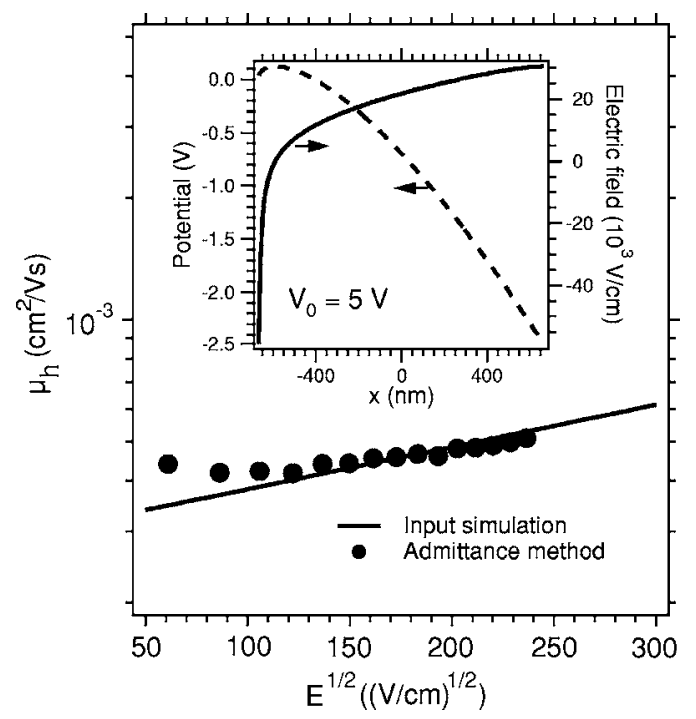

FIG. 11. Hole mobility $\mu_{h}$ in $\alpha$-NPD as function of the applied electric field $E$, determined by numerical simulation. The straight solid line is the input data of the calculations, the full circles represent the values obtained by applying the admittance method. The inset shows the electric potential and field distributions in the simulated device for $V_{0}=5 \mathrm{~V}$. 
frequency dependence of $C$ in the LF region, which governs the shape of the differential susceptance curves leading to the mobility values, can be explained at the microscopic level by the analysis of the steady-state and small-signal charge concentrations and current densities. The inductive contribution to the capacitance is due to the modulation of the hole concentration while the traps and the injected electrons are responsible for the low-frequency behavior.

\section{CONCLUSIONS}

We have determined experimental voltage-dependent admittance curves for the ITO/ $\alpha$-NPD/Al structure where the organic layer was grown by thermal evaporation. The differential susceptance curves, related to the capacitance response of the system, show for dc bias values from $V_{0}=3 \mathrm{~V}$ a dominant peak which moves to higher frequencies with increasing voltage, as for the dip in the conductance response. The lowfrequency region of the capacitance-frequency curves presents a large decreasing signal, the shape of which is shifted to higher frequencies when the applied bias increases. The HF limit of $C$ corresponds to the dielectric capacitance defined by the geometrical characteristics of the device.

The theoretical analysis is based on a numerical simulation of the whole structure containing the two electrodes and the hole conduction through the organic material. It includes the dependence of mobility on applied electric field, following Poole-Frenkel's expression. Mobility is not therefore assumed constant with $x$. A trap density of states distribution of Gaussian shape is explicitly involved in the calculations by means of a rate equation. The numerical results explain the basic mechanisms responsible for the frequency and voltage dependence of the small-signal electrical characteristics, leading to an explanation of the experimentally observed facts.

Based on the formal developments used for the simulation of electronic devices made of inorganic semiconductors, the analysis suggests that only a complete numerical study allows a correct interpretation of the admittance curves. In particular, from a detailed study of the microscopic quantities such as the steady-state and small-signal amplitudes of the hole concentration, we showed that the negative contribution to the capacitance in the IF region originates from the modulation of the majority carriers near the anode contact. Furthermore, the role of defects, which are virtually present in all real devices, cannot be neglected in the resulting ac electrical characteristics. Our investigation of the trap effect led to the formulation of the conditions under which the admittance method can be applied to the determination of carrier mobility: a negative variation of the capacitance with respect to the geometrical limit must be present, the concentration of the trap levels has to be small enough to preserve the minimum in the $C-f$ curves, and the transition frequency of traps must be lower by at least two decades than the reciprocal carrier transit time. Moreover, we have shown that electron injection must adequately be taken into account as this phenomenon can drastically modify the frequency-dependent admittance curves. Even for materials where carrier mobilities differ by several orders of magnitude, such as $\alpha$-NPD, the modulation of the minority carrier concentration, contributing to the amplitude of the variation of the total space charge, can lead to strong negative values of the measured capacitance at low frequency. This feature is governed by the strength of the recombination rate.

As demonstrated by the correlation between theoretical and experimental results, this work contributes to the basic understanding of the capacitance variations with the modulation frequency; it can be useful for the methodology of obtaining mobilities from admittance measurements. Once the system has been defined, it is possible to numerically solve the set of physical equations without any further approximation. The analysis of the literature shows that, in the past, the procedure used was certainly correct, but several steps were made on a rather empirical basis. As an example, in our interpretation, the low-frequency behavior of the capacitance can be explained by a contribution of the defect band response and the response of electrons. It is not therefore necessary to introduce frequency dependence of the carrier mobility or spectral dispersion of the transit time.

The approach developed in this paper could be applied to other organic materials. However, in order to extract mobility data from the analysis of the admittance curves, the abovementioned conditions must be fulfilled. The literature on the applicability of admittance spectroscopy to the evaluation of mobility in small-molecule organic materials is indeed much less abundant than for conjugated polymer-based devices. The experimental aspects could take into account modified conditions such as transient excitation. Finally, the numerical analysis of different continuous distribution functions of the trap density of states or the inclusion of two or more levels is also possible. The influence of the traps located near the metal-organic interface on the carrier injection mechanism ${ }^{31,41-43}$ could also be investigated by numerical simulation of the small-signal ac characteristics.

\section{ACKNOWLEDGMENT}

Financial support by INTERREG III Project No. EMR INT III 0304-2.1.57 is gratefully acknowledged.

\footnotetext{
*Author to whom correspondence should be addressed. The author is now at IMEC, Kapeldreef 75, B-3001, Leuven, Belgium. Electronic address: Duy.Nguyen@imec.be

${ }^{1}$ L. Pautmier, R. Richter, and H. Bässler, Synth. Met. 37, 271 (1990).
}

\footnotetext{
${ }^{2}$ H. Bässler, Phys. Status Solidi B 175, 15 (1993).

${ }^{3}$ H. C. F. Martens, P. W. M. Blom, and H. F. M. Schoo, Phys. Rev. B 61, 7489 (2000).

${ }^{4}$ Y. Roichman and N. Tessler, Synth. Met. 135, 443 (2003).

${ }^{5}$ W. F. Pasveer, J. Cottaar, C. Tanase, R. Coehoorn, P. A. Bobbert,
} 
P. W. M. Blom, D. M. de Leeuw, and M. A. J. Michels, Phys. Rev. Lett. 94, 206601 (2005).

${ }^{6}$ C. Tanase, E. J. Meijer, P. W. M. Blom, and D. M. de Leeuw, Phys. Rev. Lett. 91, 216601 (2003).

${ }^{7}$ E. Lebedev, Th. Dittrich, V. Petrova-Koch, S. Karg, and W. Brütting, Appl. Phys. Lett. 71, 2686 (1997).

${ }^{8}$ A. J. Campbell, D. D. C. Bradley, and H. Antoniadis, J. Appl. Phys. 89, 3343 (2001).

${ }^{9}$ M. Abkowitz, J. S. Facci, and J. Rehm, J. Appl. Phys. 83, 2670 (1998).

${ }^{10}$ D. Poplavskyy and J. Nelson, J. Appl. Phys. 93, 341 (2003).

${ }^{11}$ S. Karg, V. Dyakonov, M. Meier, W. Riess, and G. Paasch, Synth. Met. 67, 165 (1994).

${ }^{12}$ D. J. Pinner, R. H. Friend, and N. Tessler, J. Appl. Phys. 97, 014504 (2004).

${ }^{13}$ P. W. M. Blom, M. J. M. de Jong, and M. G. van Munster, Phys. Rev. B 55, R656 (1997).

${ }^{14}$ L. Bozano, S. A. Carter, J. C. Scott, G. G. Malliaras, and P. J. Brock, Appl. Phys. Lett. 74, 1132 (1999).

${ }^{15}$ H. C. F. Martens, H. B. Brom, and P. W. M. Blom, Phys. Rev. B 60, R8489 (1999).

${ }^{16}$ P. W. M. Blom, H. C. F. Martens, and J. N. Huiberts, Synth. Met. 121, 1621 (2001).

${ }^{17}$ S. Berleb and W. Brütting, Phys. Rev. Lett. 89, 286601 (2002).

${ }^{18}$ H. H. P. Gommans, M. Kemerink, G. G. Andersson, and R. M. T. Pijper, Phys. Rev. B 69, 155216 (2004).

${ }^{19}$ H. H. P. Gommans, M. Kemerink, and R. A. J. Janssen, Phys. Rev. B 72, 235204 (2005).

${ }^{20}$ S. W. Tsang, S. K. So, and J. B. Xu, J. Appl. Phys. 99, 013706 (2006).

${ }^{21}$ J. Shao and G. T. Wright, Solid-State Electron. 3, 291 (1961).

${ }^{22}$ H. C. F. Martens, J. N. Huiberts, and P. W. M. Blom, Appl. Phys. Lett. 77, 1852 (2000).

${ }^{23}$ H. C. F. Martens, W. F. Pasveer, H. B. Brom, J. N. Huiberts, and P. W. M. Blom, Phys. Rev. B 63, 125328 (2001).

${ }^{24}$ D. Poplavskyy and F. So, J. Appl. Phys. 99, 033707 (2006).
${ }^{25}$ A. B. Walker, A. Kambili, and S. J. Martin, J. Phys.: Condens. Matter 14, 9825 (2002).

${ }^{26}$ S. Selberherr, Analysis and Simulation of Semiconductor Devices (Wiley, New York, 1981).

${ }^{27}$ J. Shen and J. Yang, J. Appl. Phys. 83, 7706 (1998).

${ }^{28}$ E. Tutis, M. N. Bussac, B. Masenelli, M. Carrard, and L. Zuppiroli, J. Appl. Phys. 89, 430 (2001).

${ }^{29}$ C. D. J. Blades and A. B. Walker, Synth. Met. 111-112, 335 (2000).

${ }^{30}$ B. Ruhstaller, T. Beierlein, H. Riel, S. Karg, J. Campbell Scott, and W. Riess, IEEE J. Sel. Top. Quantum Electron. 9, 723 (2003).

${ }^{31}$ G. Paasch, A. Nesterov, and S. Scheinert, Synth. Met. 139, 425 (2003).

${ }^{32}$ N. Tessler, D. J. Pinner, and R. H. Friend, Synth. Met. 111-112, 269 (2000).

${ }^{33}$ D. J. Pinner, R. H. Friend, and N. Tessler, J. Appl. Phys. 86, 5116 (1999).

${ }^{34}$ M. A. Lampert and P. Mark, Current Injection in Solids (Academic, New York, 1970).

${ }^{35}$ D. Poplavskyy, W. Su, F. Pschenitzka, and F. So, Proc. SPIE 5519, 110 (2004).

${ }^{36}$ S. Naka, H. Okada, H. Onnagawa, Y. Yamaguchi, and T. Tsutsui, Synth. Met. 111-112, 331 (2000).

${ }^{37}$ M. Schmeits and N. D. Nguyen, Phys. Status Solidi A 202, 2764 (2005).

${ }^{38}$ N. D. Nguyen and M. Schmeits, Phys. Status Solidi A 203, 1901 (2006).

${ }^{39}$ E. H. Rohderick and R. H. Williams, Metal-Semiconductor Contacts (Clarendon, Oxford, 1988).

${ }^{40}$ M. Sakhaf and M. Schmeits, J. Appl. Phys. 80, 6839 (1996).

${ }^{41}$ M. A. Baldo and S. R. Forrest, Phys. Rev. B 64, 085201 (2001).

${ }^{42}$ T. Lindner, G. Paasch, and S. Scheinert, J. Mater. Res. 19, 2014 (2004).

${ }^{43}$ S. J. Konezny, D. L. Smith, M. E. Galvin, and L. J. Rothberg, J. Appl. Phys. 99, 064509 (2006). 